\title{
A Study On Graph Theory Properties In Human Blood Circulatory System
}

\author{
R.B.A.H. Abeyrathne and G.H.J. Lanel \\ Department of Mathematics, University of Sri Jayewardenepura, Sri Lanka. \\ DOI: 10.29322/IJSRP.11.10.2021.p11851 \\ http://dx.doi.org/10.29322/IJSRP.11.10.2021.p11851
}

\begin{abstract}
Network science and graph theory applications have spread widely to help in understanding how human body features are linked to graph network structure, thus providing a conceptual framework that can help in reducing the analytical circulatory complexity and underlining how network topology can be used to characterize circulatory diseases [1]. The central goal of this study was to verify the possibility of creating a structural network representing the human blood circulatory system and to describe the relationship between the human bodies in graph theory basics. Graph theory concepts were applied to 6 organs which contain selected 162 body parts and 217 links, representing the blood circulatory system. The open-source platform GEPHI was used to obtain graph models. In addition, the "NetworkAnalyzer" plugin was used to get the graph theory results. To achieve more results of network parameters Maple Software was used. At the results of this study, the shortest pathways, network diameter, network radius, graph density, modularity, spanning trees, clusters, and connected components were obtained according to the 6 main organs in the modeled human blood circulatory system using the "NetworkAnalyzer" plugin. Moreover, clique number, chromatic number, regularity, planetary, hamiltonicity, eulericity, and the number of cycles were obtained by Maple.
\end{abstract}

Index Terms - Anatomical relationships, blood vessels, graph network model, human blood circulatory system, and main organs.

\section{INTRODUCTION}

Graph theory is one of the most important branches of mathematics particularly discrete mathematics which is also called the mathematics of network. For the past two decades graph theory is playing a vital part in image segmentation techniques which is the most active research topic nowadays. In this study, we have presented the techniques of graph theory and developed a model for a micro cardiac network system. The main concept is to get the blood flow in the human body concerning oxygenated and deoxygenated blood circulation using the graph theory properties [2]. This report utilizes network analysis by focusing on the visualization of the circulatory system using GEPHI Software. This work will help to develop a tool for solving the blood flow system in the human body.

\section{Human Blood Circulatory System}

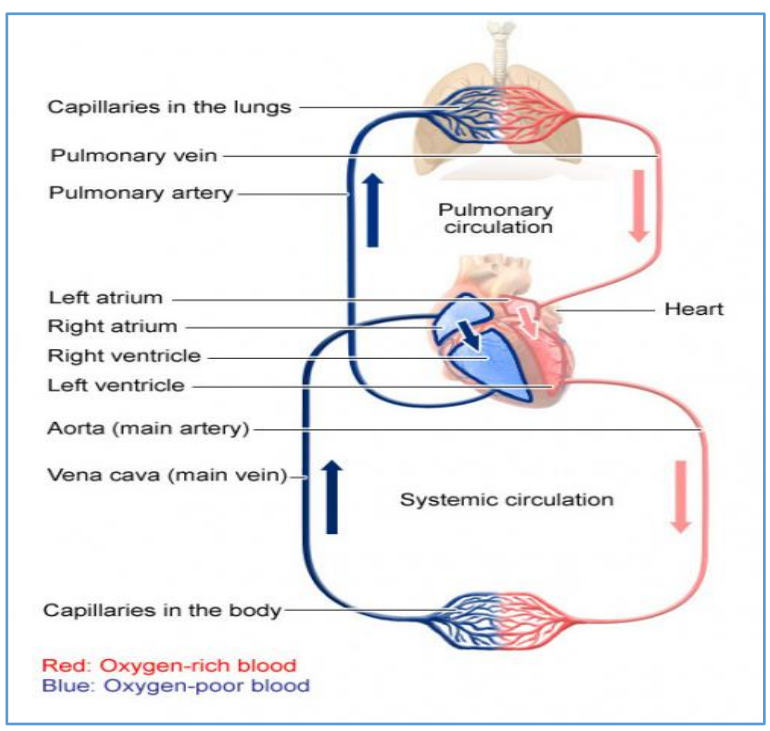

The blood circulatory system consists of the heart and all the blood vessels that run throughout the entire body. It distributes nutrients and oxygen to all cells in the human body. The arteries carry blood off from the heart and the veins carry it back to the heart. The system of blood vessels bear resembles a tree: The main artery (aorta) - branches into large arteries, which lead to smaller and smaller vessels. The smallest arteries end in a network of tiny vessels known as the capillaries and the network of tiny veins starts from it. The systemic circulation supplies organs, tissues, and cells with blood so that they get oxygen and other vital substances. Pulmonary transmission is where the fresh oxygen we breathe in enters the blood. At the same time, when we breathe out, carbon dioxide is free from the blood [3]. 
Figure 1: Structure of Human Blood Circulatory

System.

\section{Graph Theory Approach to Neuroscience}

The main ability of graph theory in neuroscience studies is usually exposed after the construction of a functional brain network. Several graph theory results can be used to assess the topological patterns of different networks. Typically, one cannot claim which measures are more suitable for studying the brain network [4], but given the detailed structure of the human brain, measures that can represent the small-world properties of the brain network are of huge importance. Moreover, in recent years the human body and the relations between its different parts have been studied using the theory of networks [4].

This approach has allowed the study of the human body from a different perspective. The results achieved by the structured network representing the musculoskeletal system can be further studied and graphically represented with the concepts of the graph theory. In this sense, graph theory is applied to several fields, including the medical and social ones [4].

\section{MATERIALS AND METHODOLOGY}

There are 3 main blood vessels. Arteries carry oxygenated blood away from the heart, Veins carry blood back to the heart, and capillaries connect tiny arteries and veins. Some blood vessels have more than $20 \mathrm{~mm}$ diameter while some small blood vessels have micrometer diameters. Therefore specifically major blood vessels which have more than $2 \mathrm{~mm}$ average diameter in the brain, neck, heart, hands, digestive system, urinary system, and legs were considered according to the "Medical Physiology, 3rd Edition, Elastic Properties of Blood Vessels". Among these structures (nodes), the directed links (the connection is associated with a direction) have been defined according to the existing anatomical relationship.

Therefore as a whole, we considered a total of 162 anatomical structures or nodes in the above 6 main organs and 217 connections among those anatomical structures. Moreover, instead of the above organs, all the graph-theoretic statistics were reobtained for the upper limb which includes the heart, neck, head, hands, and digestive system together, and for the lower limb which includes the urinary system and legs together [5].

In this sense, the open-source software platform GEPHI (www.GEPHI.org) was used for data entry (nodes and links). In addition, the plugin "Network Analyzer" was used for the graph theory outputs like shortest path, spanning tree, density, modularity, average degree, diameter, and radius and clustering coefficient of the network obtained. The resulting network parameters were then imported for a better rendering, using the plugin "Force Atlas 2 and no overlap". The structured network models obtained and expressed through a graph were characterized by several attributes like: between centrality, closeness centrality, bridging centrality, degree, eccentricity, and modularity classes.

Moreover for some graph theory statistics which are not given by "NetworkAnalyzer" such as a number of cycles, Hamiltonian cycles, Euler circuits, clique number, chromatic number, and planer graphs were obtained using Maple software for every 6 main organs. The GraphTheory package in Maple which supports both directed or undirected graphs and weighted or unweighted graphs was used.

\section{VISUALIZATION OF GRAPH MODELS}

According to the above process following graph network models were obtained for the human body. Here red color indicates arteries, blue color for veins and yellow indicates capillaries. The size of the node was ranked according to the closeness centrality with the size scale is 80- 160 for the perfect visualization. Contraction, Expansion, Nonoverlap, and Label adjust; are the 4 main layouts that were used. The scale factor is 0.8. After that using the Network Analyzer plugin all the graph theory results were obtained.

\section{Graph Model for Human Heart}




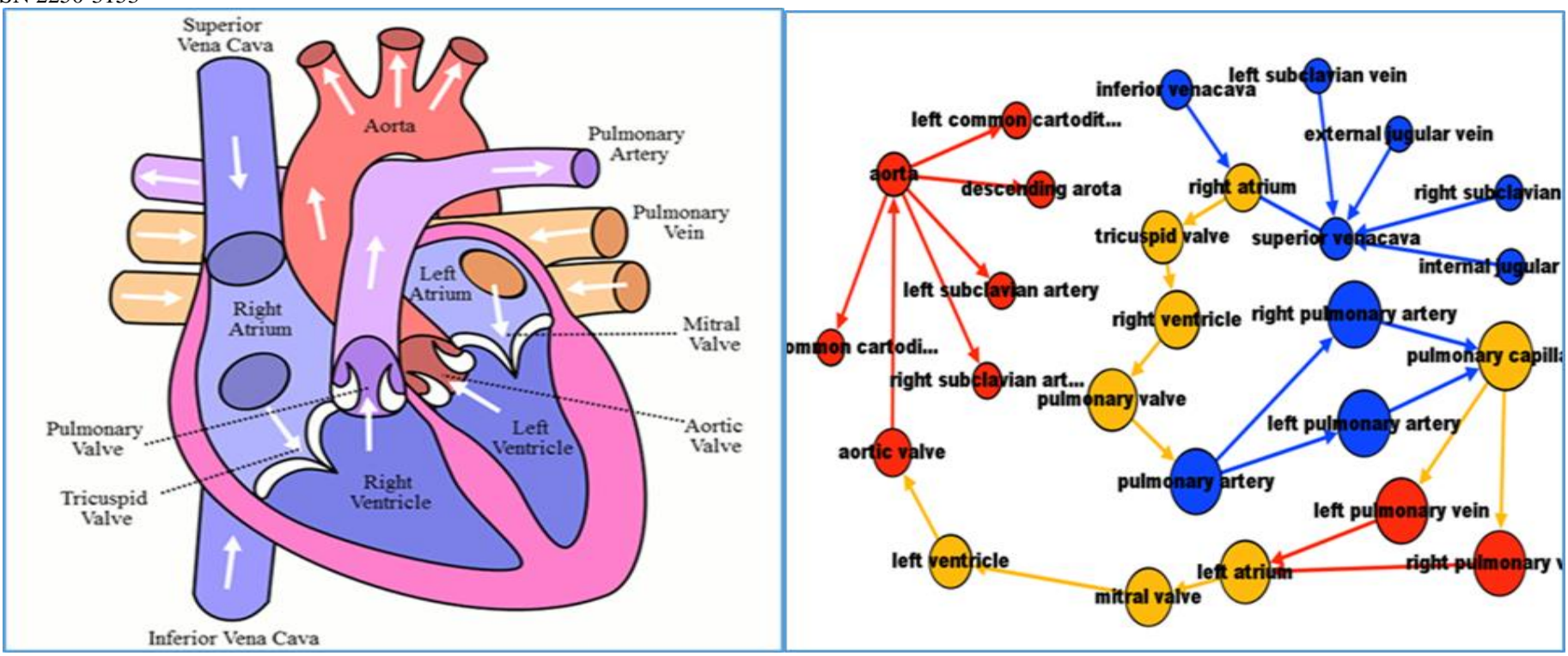

Figure 2: Human heart anatomy [2] vs heart graph network model

The human heart is a muscular organ that is about the size of a closed palm that performs the pumping function of the body's blood circulatory system. Aorta, the largest artery in the body is in the heart [2]. In the model, there are 26 nodes and 27 edges. Here all the blood vessels including capillaries are also considered as nodes and edges have the source node color.

\section{Graph Model for Human Neck and Brain}

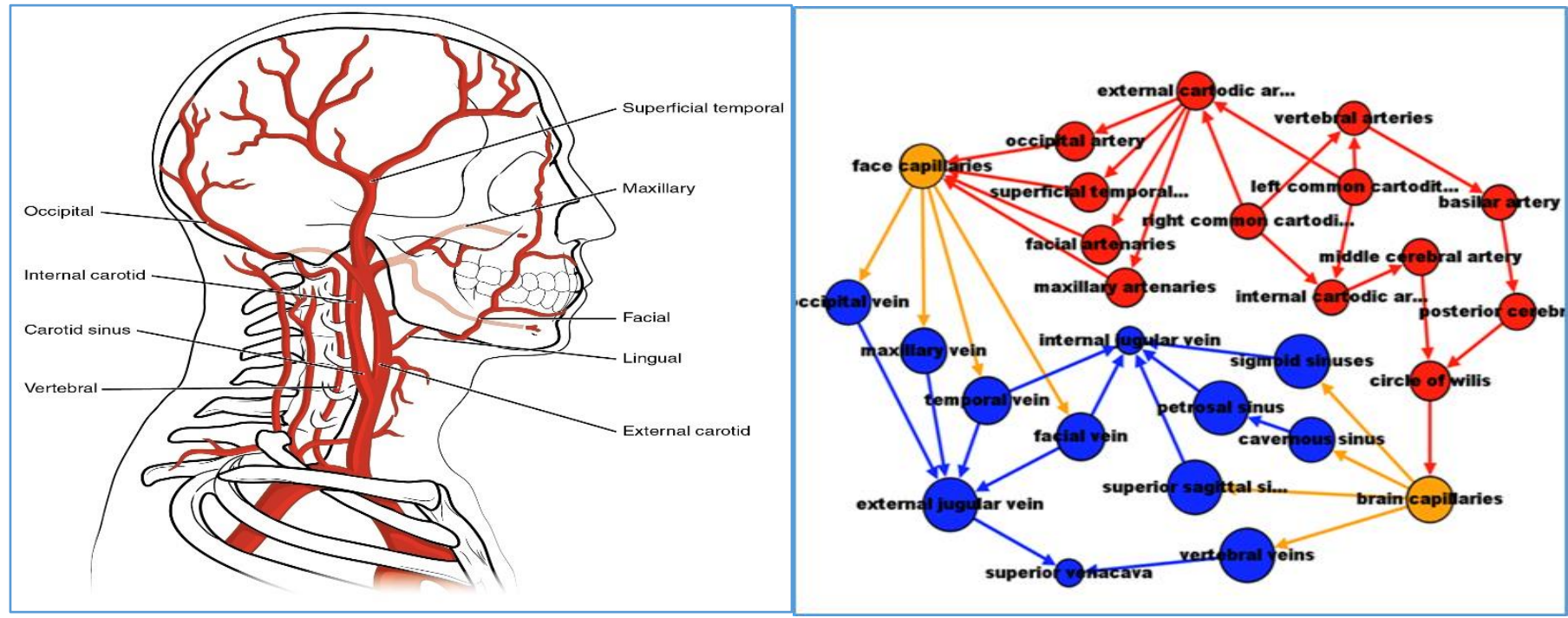

Figure 3: Arteries of neck and brain [11] vs graph network model

Blood is donated to the neck, head, and brain through subclavian and common carotid arteries. The vertebral arteries are a main division of the subclavian artery. The internal carotid artery passes through the temporal bone of the skull to supply oxygenated blood to the parts of the brain and face. The external carotid artery is divided into branches that supply the skin and muscles of the face, side, and back of the head respectively [6].

The external jugular veins and internal jugular veins lie superficial to them. They receive blood from the regions of the face, scalp, and neck. The vertebral veins descend from the transverse openings of the cervical vertebrae and enter the subclavian veins. In the graph network model, altogether there are 27 selected main blood vessels (nodes) and 41 edges as above mentioned. A special feature called the Circle of wills in the brain can be easily recognized.

\section{Graph Model for Human Hands}

This publication is licensed under Creative Commons Attribution CC BY. 


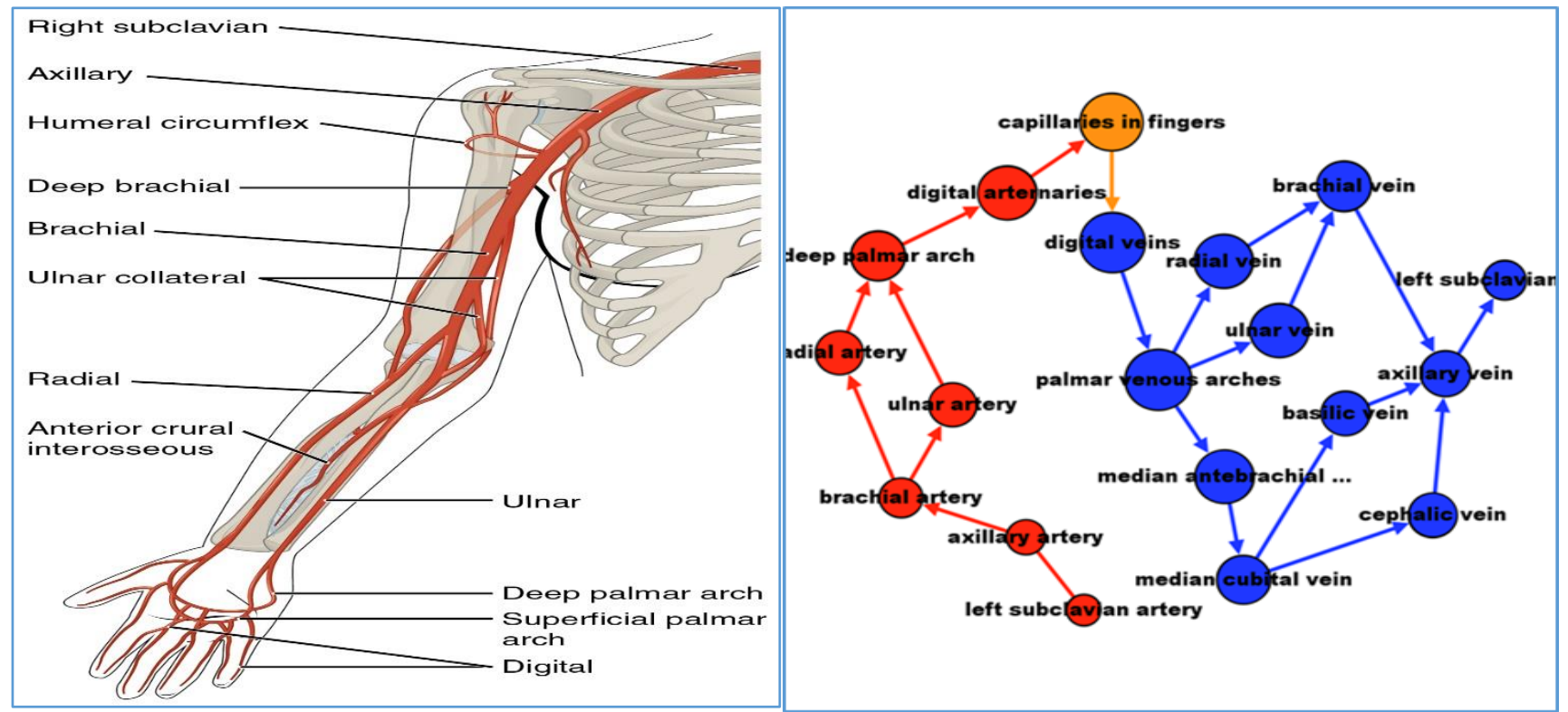

Figure 4: Major arteries serving human hands [11] vs graph network model

Oxygenated blood enters the arm by leaving the aortic arch and flowing into subclavian arteries. From here, blood flows through the brachial artery and into the radial or ulnar arteries. They continue down the arm and through the wrist before connecting into the deep palmar arch. This arch is a combination of arteries formed at the joint of the ulnar and radial arteries in the palm. This semicircular artery branch goes into the fingers, where its divisions are known as palmar digital branches.

On the top of the hand, the dorsal venous is a group of veins that spreads across the back of the hand to return blood to the heart and can be pumped to the lungs and become replenished with oxygen. To get from the hand to the heart, the deoxygenated blood flows up the shoulder area through either the basilic or cephalic veins. In the graph model, there are mainly 19 nodes and 22 edges as mentioned above.

\section{Graph Model for Human Digestive System}

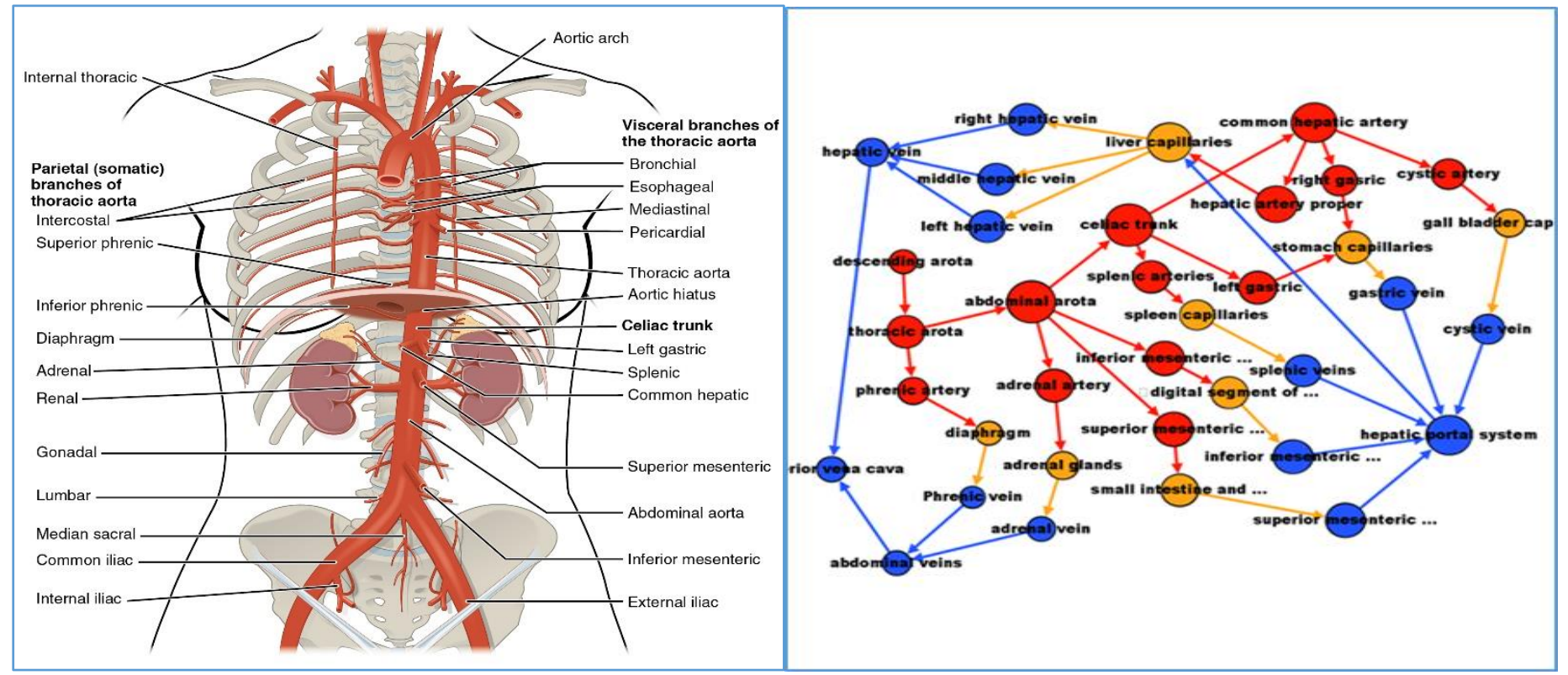

Figure 5: Arteries of the digestive regions [11] vs graph network model

Some branches of the celiac trunk carry arterial blood to the stomach. The celiac trunk is a short, broad artery that starts from the abdominal portion of the aorta, which is the main vessel conveying arterial blood from the heart to the overall systemic circulation. Blood from the stomach is entered into the venous system through the portal vein, which carries the blood to the liver.

This publication is licensed under Creative Commons Attribution CC BY. 
In the graph model, there are 36 blood vessels as node-set and 45 edges among them. There is one special feature that calls the hepatic portal system which starts from capillaries and ends in capillaries also [7].

\section{Graph Model for Human Urinary System}

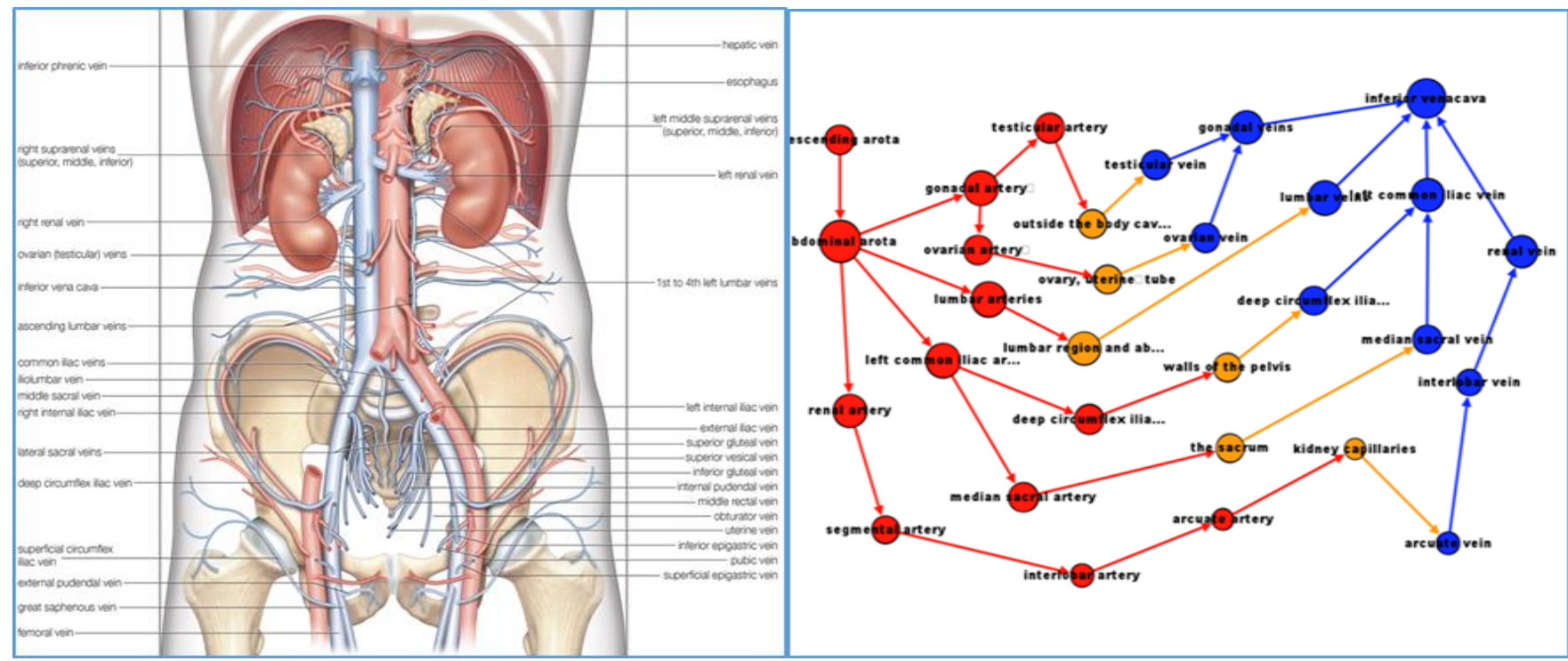

Figure 6: Human urinary system anatomy [7] vs graph network model

The urinary system contains all the organs involved in the construction and release of urine. It comprehends the kidneys, ureters, bladder, and urethra. The kidneys are bean-shaped organs that help the body make urine to get rid of unwanted misuse of substances. They balance the level of water in the body. The main function of the bladder, which is pyramid-shaped in the pelvis is to store urine and, under the appropriate signals, release it into a tube that carries the urine out of the body. The male urethra is provided by the inferior vesical and middle rectal arteries. The veins follow these blood vessels. [7]

In the graph model, we consider the male urinary system. If it is the female urinary system the difference is having ovarian veins and arteries instead of testicular veins and arteries. There are altogether 30 nodes and 34 connections between them.

\section{Graph Model for Human Legs and Feet}

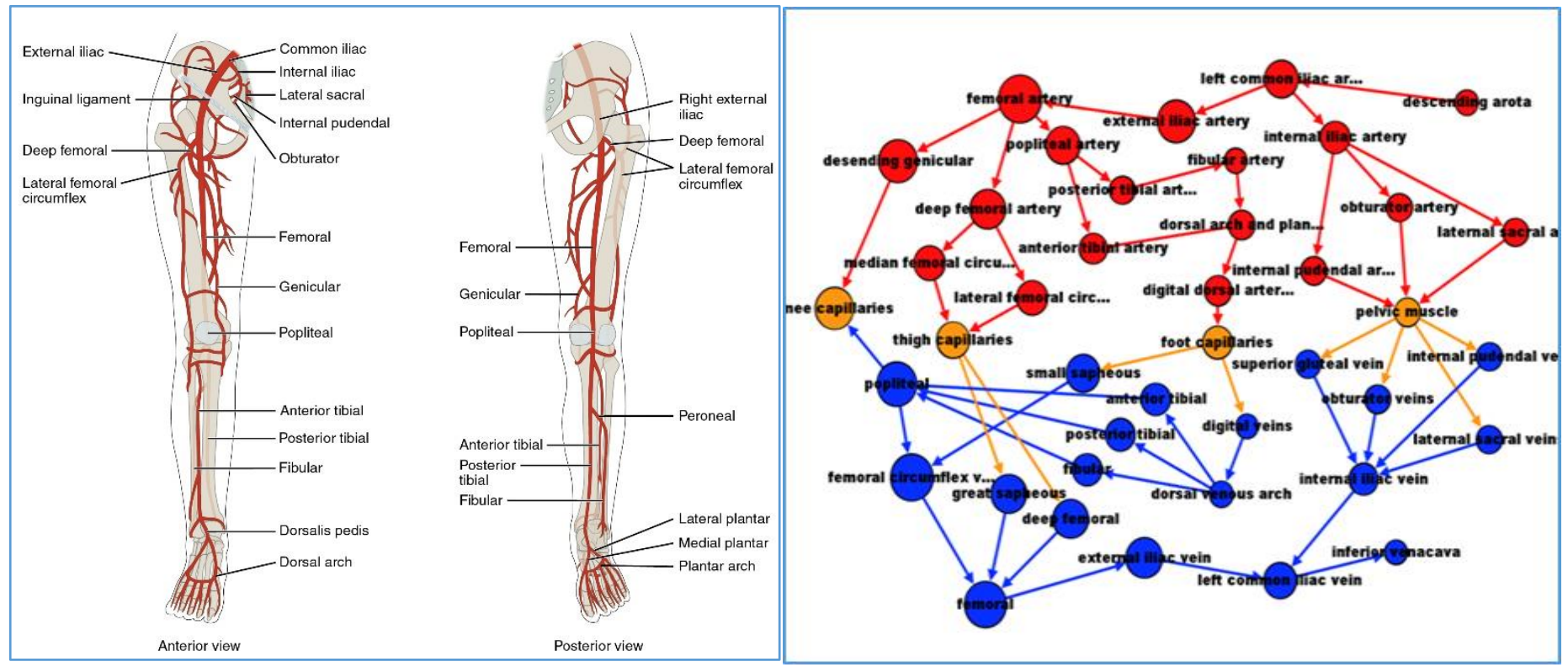

Figure 7: Major Arteries Serving human legs and feet [11] vs graph network model 
Below the kidneys, the abdominal aorta splits into two main branches near the abdomen. These are called the common iliac arteries. One goes down each leg and branches into internal and external iliac arteries, which carry blood to other branches, including the femoral artery. The femoral artery, the major artery in the thigh, continues to branch into other smaller arteries as blood travels down to the tips of the toes. The popliteal artery is a branch of the femoral artery, it supplies blood to the knee, thigh, and calf. It stops at the anterior and posterior tibial arteries. The posterior tibial artery is a branch of the popliteal artery that supplies oxygenated blood to the leg and sole. It runs on the inner of the leg and is accompanied by the posterior tibial vein. The anterior tibial artery is the other branch of the popliteal artery from the back of the knee, it supplies blood to the muscles of the leg and foot. The plantar arteriesateral, medial, and deep form a looping web across the foot and down through each toe and unite with the dorsalis pedis artery.

The dorsal pedis vein accompanies this artery. With an opposite function to arteries, veins return deoxygenated blood to the heart, where it will begin the journey all over the body again. Often, veins run the same structure as arteries. Some of them are the internal and external iliac veins, femoral vein, saphenous vein, popliteal vein, tibial vein, and the venous arch of the foot. For the graph model including these vessels, we considered 41 blood vessels as the node-set and 54 connections between them for the left leg.

\section{RESULTS AND DISCUSSION}

This analysis is based on the results obtained from "GEPHI" software using the "NetworkAnalyzer" plugin for all the 6 main organs in the human blood circulatory system and then for the upper limb, lower limb, and full body. All the quantitative graph theory outputs are given below in the table.

Table 1: Numerical outputs for 6 main organs, Upper limb, Lower limb, and Whole-body from GEPHI

\begin{tabular}{|c|c|c|c|c|c|c|c|c|c|}
\hline & Heart & $\begin{array}{l}\text { Neck } \\
\& \text { Head }\end{array}$ & Hands & Digestive & Urinary & Legs & $\begin{array}{l}\text { Upper } \\
\text { Limb }\end{array}$ & $\begin{array}{l}\text { Lower } \\
\text { Limb }\end{array}$ & $\begin{array}{l}\text { Full } \\
\text { Body }\end{array}$ \\
\hline Number of Nodes & 26 & 27 & 19 & 36 & 30 & 41 & 64 & 97 & 160 \\
\hline Number of Edges & 27 & 41 & 22 & 45 & 34 & 54 & 87 & 128 & 217 \\
\hline Ave. Degree & 2.077 & 1.519 & 2.316 & 1.250 & 1.133 & 2.634 & 1.359 & 1.320 & 1.356 \\
\hline Network Diameter & 15 & 6 & 12 & 8 & 7 & 10 & 14 & 12 & 16 \\
\hline $\begin{array}{l}\text { Network } \\
\text { Radius } \\
\end{array}$ & 8 & 5 & 6 & 6 & 6 & 7 & 10 & 8 & 11 \\
\hline $\begin{array}{l}\text { Graph } \\
\text { Density }\end{array}$ & 0.083 & 0.057 & 0.129 & 0.036 & 0.039 & 0.066 & 0.043 & 0.013 & 0.017 \\
\hline $\begin{array}{l}\text { Ave. Clustering } \\
\text { Coefficient }\end{array}$ & 0 & 0 & 0 & 0 & 0 & 0 & 0 & 0.007 & 0.003 \\
\hline $\begin{array}{l}\text { Ave. Path } \\
\text { Length }\end{array}$ & 6.649 & 2.813 & 4.614 & 3.871 & 4.082 & 4.847 & 6.321 & 5.862 & 7.169 \\
\hline Modularity & 0.617 & 0.481 & 0.549 & 0.556 & 0.558 & 0.634 & 0.690 & 0.694 & 0.763 \\
\hline $\begin{array}{l}\text { No. of edges Spanning } \\
\text { Tree }\end{array}$ & 25 & 26 & 18 & 35 & 29 & 40 & 63 & 96 & 159 \\
\hline $\begin{array}{l}\text { Number of modularity } \\
\text { clusters }\end{array}$ & 5 & 4 & 4 & 5 & 5 & 5 & 7 & 9 & 10 \\
\hline
\end{tabular}

\section{Interpretation of Graph Theory Findings Obtained from GEPHI Software}

Each of these measures provides a statistical output that needs to be interpreted to understand each network and to be able to make accurate comparisons between graph networks. Fortunately, many of these measures provide normalized output, that is, values lie between zero and one, facilitating easy comparisons between different graph networks.

\section{Diameter and Radius Interpretation}


Diameter, which is merely a measure of the distance between the two most distant nodes in the network. Let's consider two networks, each with a diameter of six. Simple, right? At least until we say that Network $A$ has 200 nodes, while Network $B$ has 2,000. This begins to alter our perception of the structure of each network relative to the other. In this study according to the table least number of nodes are in the hands and their diameter is 12 on the other hand highest number of nodes in the whole body and its diameter is 14 . Apart from that heart has only 26 nodes but the diameter is 15 . It is a relatively large value according to the other organs. Radius is the minimum among all the maximum distances between pair of nodes. Similarly, the whole body has the highest radius and it is 11 [8].

\section{Graph Density Interpretation}

It gives critical feedback on the structure of the graph as measured by the proportion of edge connections relative to the total available number. According to this study highest graph density was obtained for hands and the lowers density was obtained for lower limbs of human anatomy.

\section{Average path-length interpretation}

It can provide insight into the general structure and connectedness of a network. If we reference the average path length for a graph and find it to be very close to the network diameter, then this is an indication that the graph is inefficient from a communication standpoint. If this is the case, it may well be due to the presence of multiple clusters in the network that add friction to the process of traversing the graph. On the other hand, if the Average Path Length measure is well below the diameter, then it is likely that the network is efficient, and information can easily flow across the graph. In this study, the average path length outputs in the table for all the organs in human anatomy were below the diameter [8].

\section{Clustering coefficient Interpretation}

The global clustering coefficient is based on the concept of triplets: three nodes often close to one another, connected by two or three edges. In the case of two connections, the triplet is referred to as an open triplet, while if all three nodes are connected, we have a closed triplet, also referred to as a triangle. The derived statistic is simply calculated by dividing the number of closed triplets by the total number of triplets (open and closed), resulting in a coefficient value between 0 and 1 . Although for all the 6 main organs in the human body clustering coefficient is zero. That means no closed triplets in blood vessels in each organ [8].

\section{The Modularity statistic Interpretation}

It places individual nodes into an aggregated group or cluster based on shared characteristics. This is a simpler approach compared to plugins expressly designed for clustering and partitioning, but it can nonetheless help in understanding the network and provides a very easy means to do so when combined with filtering methods. Output for this function is simply an integer value starting at 0 .

\section{The number of edges in the minimum spanning tree interpretation}

This plugin calculates a minimum spanning tree for a graph using Kruskal's Algorithm. If the edge is in the minimum spanning tree, the value in the "Minimum Spanning Tree" attribute is "1", otherwise it is " 0 ". The number of edges in the minimum spanning tree is equal to the weight of the minimum spanning tree because the same weight was given to every edge. It can be visualized by creating a Filter on the Attribute "Minimum Spanning Tree" with value = "1". According to the number of edges, the highest weight minimum spanning tree is observed from the whole blood circulatory system, and the lowest weight coming under the blood vessels in hands [8].

\section{Graphical Outputs Form GEPHI}
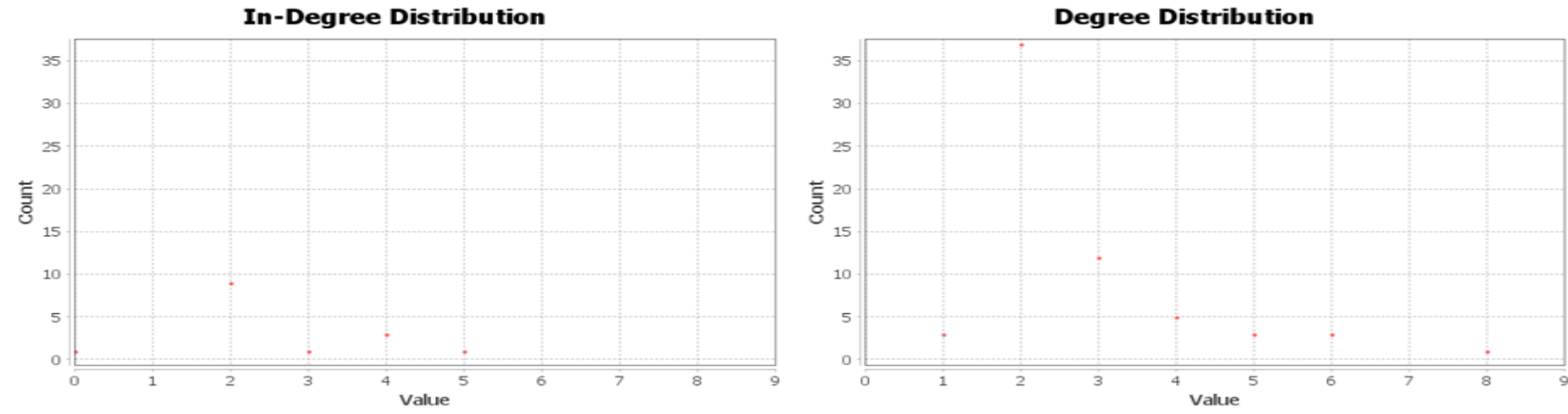

This publication is licensed under Creative Commons Attribution CC BY. 


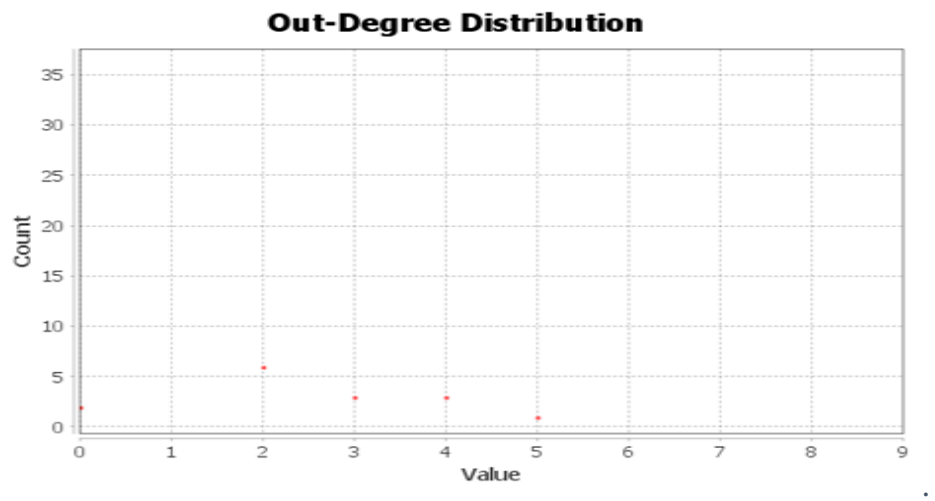

Figure 8: Degree Distribution Scatter plots of Human Upper Limbs.

These scatter plots represent the degree distribution for the network model. This type of analysis will use for understanding the network before visualization.

\section{Modularity Classes}

The number of distinct groupings within a network can be done simply by using one of the GEPHI plugins geared to parse nodes into distinct groups. The Chinese Clusters tool can be used for this purpose, as well as the MCL, MCODE, and Girvan Newman plugins. The end goal for any of these algorithms is to group nodes based on the strength of their relationships. Highly connected nodes are likely to wind up in a common cluster, regardless of which algorithm is employed. Following graphs are the 7 modularity classes of human upper limbs [8].
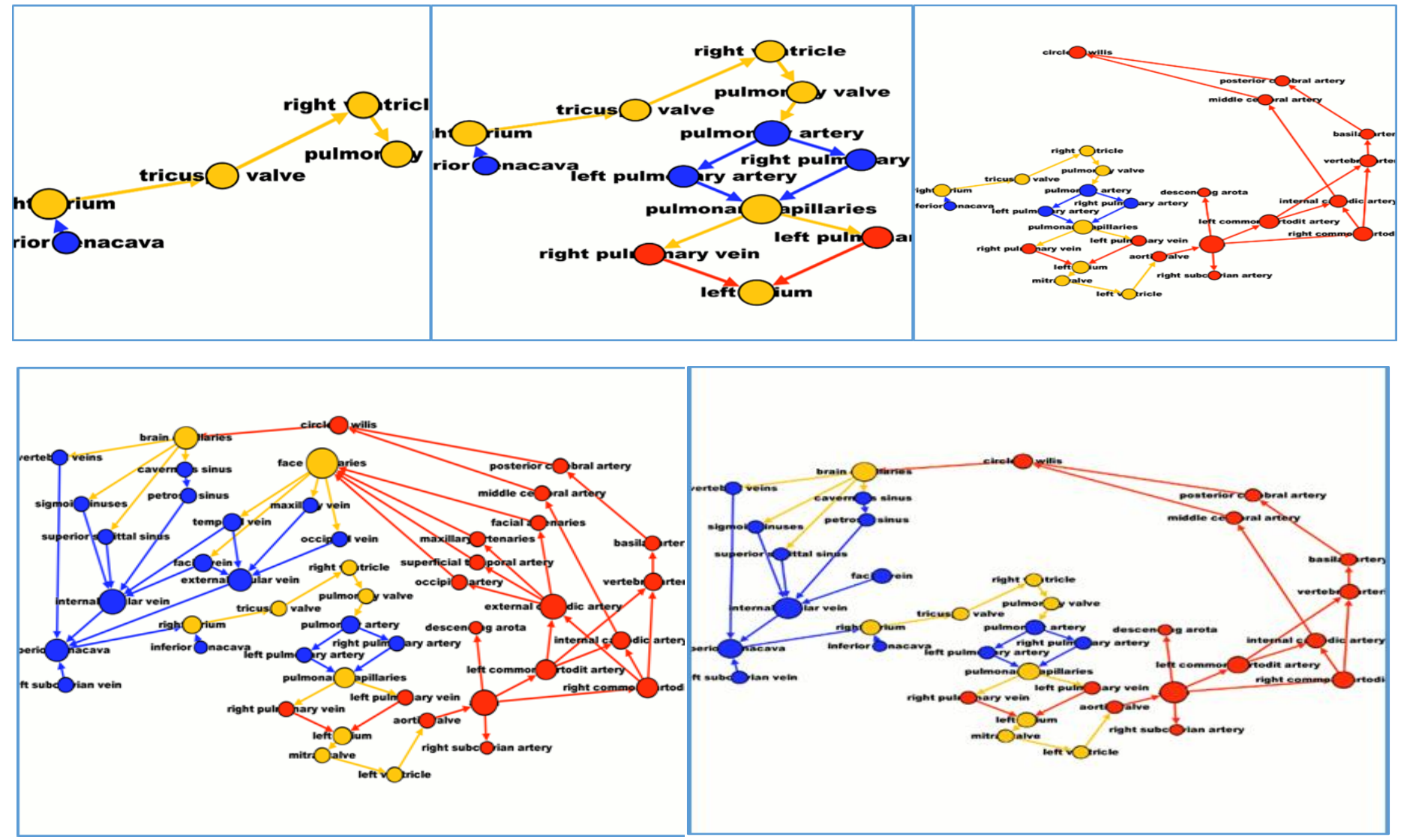


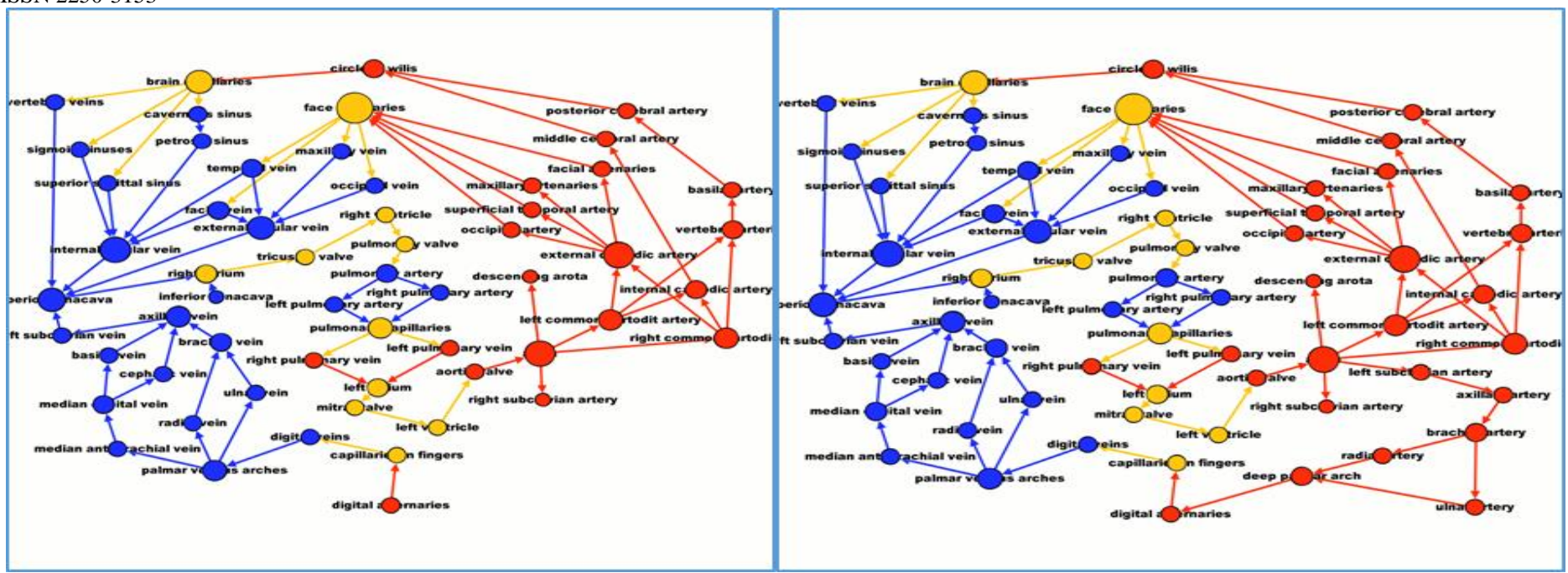

Figure 9: Modularity Classes of human upper limb

\section{Graph Distance report of human upper limbs from GEPHI}

\section{Betweenness centrality interpretation}

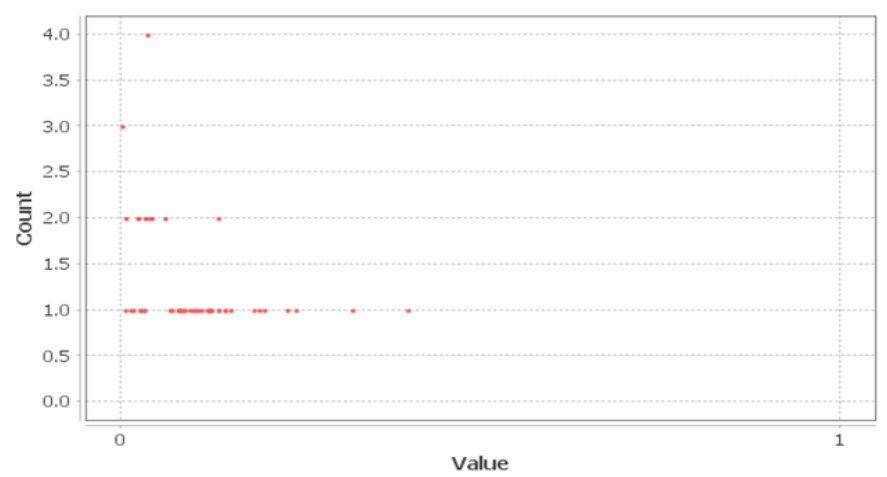

Figure 10: Betweenness Centrality Distribution of Human Upper Limbs

\section{Closeness centrality Interpretation}

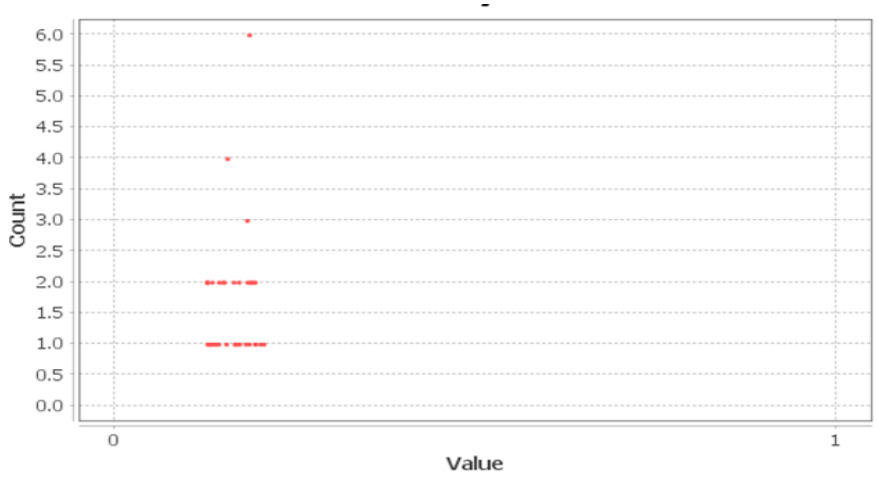

Figure 11: Closeness Centrality Distribution of Human Upper Limbs
Unlike the other centrality measures, which are largely predicated on direct connections and/or proximity to others with many connections, betweenness measures how important an individual node is for others traversing the network. Specifically, this measure tells us how frequently a given node lies down on the shortest path between two other nodes. It is quite possible, and often likely, that a member with a very high betweenness centrality score has very low numbers on all of the other centrality calculations [8], [9].

This will be highly dependent on the network structure and is far more likely to be true in networks with higher levels of clustering.

In this case, defined by the effort required (as measured by path length) to reach all other nodes in the network. As noted earlier, we might well have several graph members who score low on other measures such as degree centrality, but who still exercise influence based on their position within the network. It is often the case that a single node scores highly on each of these measures; if an individual has enough degree connections, it will likely have fewer long path connections to other nodes, resulting in a lower (that is, more connected) score on this statistic. In terms of output, each node will have a measure higher than 1 , with lower values indicating greater centrality [8], [9]. 


\section{Spanning Tree obtained from GEPHI}

A tree of graph $G$ is a connected subgraph containing no circuits. If a tree of a connected graph $G$ contains all the vertices of $G$ then it is called a spanning tree of $G$. The complement of a spanning tree $T$ in $G$ is called a Cospanning tree of $G$.

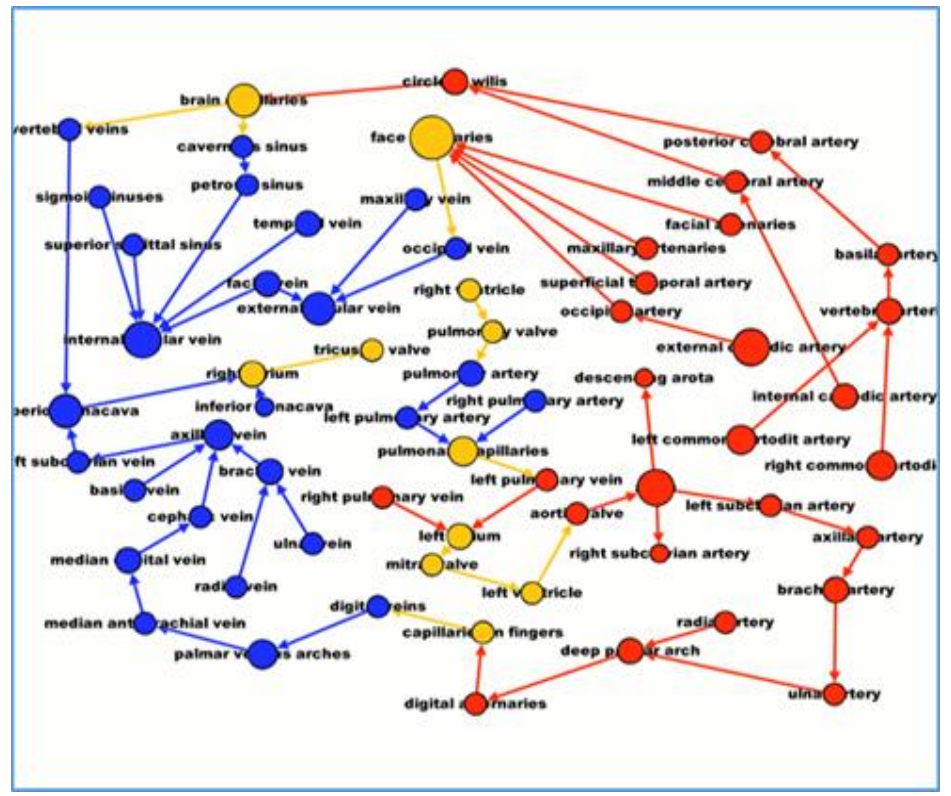

Figure 12: Spanning Tree for human upper limbs

\section{Shortest Path Interpretation}

The shortest path is a path between two vertices in a graph such that the sum of the weights of its constituent edges is minimized. Purple color displays the shortest path between any pair of nodes if at least one path exists between nodes.

Similarly, all the above outputs were obtained for the other 6 main organs and lower limbs. Moreover, remaining graph theory statistics which are not running by "Network Analyzer" were obtained using the GraphTheory package in Maple software for 6 main organs.

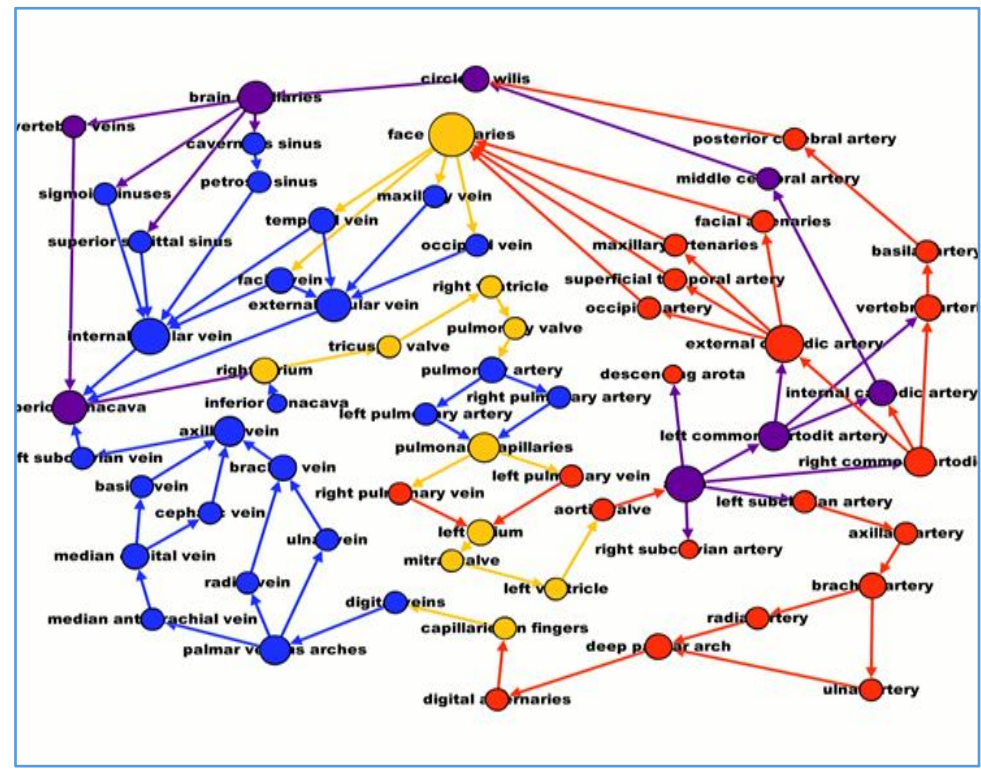

Figure 13: Shortest path between the aorta and superior venacava in human upper limbs

\section{Main Findings from Maple Software}

This publication is licensed under Creative Commons Attribution CC BY. 
Table 2: Qualitative Outputs for 6 main organs obtained from Maple Software

\begin{tabular}{|l|l|l|l|l|l|l|}
\cline { 2 - 7 } \multicolumn{1}{c|}{} & Heart & $\begin{array}{l}\text { Brain } \\
\text { Neck }\end{array}$ & Hands & Digestive & Urinary & Legs \\
\hline No. of blood vessels & 26 & 27 & 19 & 36 & 30 & 41 \\
\hline No. of connections & 27 & 40 & 22 & 44 & 33 & 54 \\
\hline Clique No. & 2 & 2 & 2 & 2 & 2 & 2 \\
\hline Chromatic No. & 2 & 3 & 3 & 3 & 2 & 3 \\
\hline Is Connected & Yes & Yes & Yes & Yes & Yes & Yes \\
\hline Is Regular & No & No & No & No & No & No \\
\hline Is Planar & Yes & No & Yes & Yes & Yes & Yes \\
\hline Is Hamiltonian & No & No & No & No & No & No \\
\hline Is Eulerian & No & No & No & No & No & No \\
\hline Is Tree & No & No & No & No & No & No \\
\hline Is Forest & No & No & N & No & No & No \\
\hline No. of Cycles & 2 & 14 & 4 & 9 & 4 & 14 \\
\hline
\end{tabular}

\section{Interpretation of Maple Outputs}

According to the maple outputs, the clique number is 2 and it is the same for all the main 6 organs in human blood circulation. That means for all maximum cliques in the maple plots of blood vessels contain only 2 vertices. The chromatic number varies between 2 and 3 for all the plots. When the vertices are much higher it goes to the value 3. All the 6 graphs are connected but none of them has the same number of neighbors. Planetary one of the main important results of the study occurs for 5 main organs instead of the human brain because it is a huge complex network. Unfortunately, all the graphs of organs failed to give at least one eulerian or Hamiltonian path. Moreover not a tree or a forest. Finally, some cycles were obtained according to the undirected graph plots using my cyclebasis command.

\section{CONCLUSION}

In this study, we created an anatomical graph network model for the human blood circulatory system by evaluating some of its anatomical structures and the relationships that every single structure (node) has with the others through the definition of edges. We carried out this study with 15 the same procedure as the previous one had done on the human locomotor system [5], and further analysis extended to a new and more detailed description of the circulation system using the graph theory.

By applying certain concepts of graph theory to the human body, it has been possible to develop a complete anatomical network model of the blood circulatory system by GEPHI Software. Then remaining outputs were obtained by maple software. At the same time, the density value of the generated network demonstrated that the network and its structure is very flexible and elastic, the clustering coefficient obtained from the analysis for this study is 0.003 , which can be considered as a lower value indicating that the network is neither random nor "strongly organized"[5]. Graph theory outputs such as node degree, clustering coefficient, average path length, density, centrality, modularity, shortest path, and diameter can be utilized to detect the topological patterns of circulatory networks.

To conclude these findings, we can draw some problems to be addressed by future research. The first important conclusion is that the theory of graph networks, which originated with the discovery of small-blood vessels pattern is a very useful framework for the study of large-scale networks in the human blood circulatory system. There are several reasons for this, the output models provide us with 
powerful realistic models of complex networks in the 6 main organs; the graph theory allows us to better understand the correlations between network structure and the real architecture of the body; different types of blood disease can disrupt the optimal small-blood vessels pattern. Some of these conclusions may provide useful starting points for future studies [1].

\section{SUGGESTED APPLICATIONS}

- Can verify the possibility of creating a structural network representing the human blood circulation system with the shape of the human anatomy and then by the similar procedure for animals.

- The application of this technique to patient data might aid in monitoring the impact of eventual pharmacological and rehabilitative treatments.

- The crossing numbers will be an important parameter than the unhealthy cerebrum and common functional ECG and MRI reports.

- The discovery of the graph planarity in the small-world pattern sometimes will be giving rise to identify, characterize, and resilient more consistent diagnosis of neurological hidden diseases in more random networks [1].

- The resulting model will be a handy tool for junior cardiologists, Physiotherapists, doctors, researchers, etc. to study the large-scale networks in the human body.

\section{REFERENCES}

[1] F. Vecchio, F. Miraglia, and P.M. Rossini, "Connectome: Graph theory application in functional brain network architecture," Clinical Neurophysiology Practice 2, pp. 206-213, 2017.

[2] B. Basavaprasad, and S. Ravindra, “A Graph Theoretical Approaches for Image Segmentation”, Journal of Avishkar - Solapur University Research Journal, vol. 2, 2012.

[3] InformedHealth.org [Internet]. Cologne, Germany: Institute for Quality and Efficiency in Health Care, "How does the blood circulatory system work?" [Up dated 2019 Jan 31].2006. [Online], Available: https://www.ncbi.nlm.nih.gov/books/NBK279250/

[4] E. Bullmore and O. Sporns, "Complex brain networks: graph theoretical analysis of structural and functional systems," Nature Reviews Neuroscience, 10(3): 186, 2009.

[5] P. Ferdinando, S. Ugo, and D. Daniele, “An Application Of The Graph Theory To The Study Of The Human Locomotor System”, Italian Journal Of Anatomy And Embryology, vol. 124, pp. 353-363, 2019.

[6] [Online] Available: https://www.doctorabel.us/anatomy-physiology/blood-vessels-of-the-head-and-neck.html

[7] Britannica, the Editors of Encyclopaedia. "Vein," Encyclopedia Britannica, 7 Aug. 2013, [Online], Available: https://www.britannica.com/science/vein-blood-vessel. Accessed 3 October 2021

[8] K. Cherven, Mastering GEPHI Network Visualization, Packt Publishing Ltd, Livery Place, 35 Livery Street, Birmingham B3 2PB, UK, 2015.

[9] H.S.S.P. Jayawardena and G.H.J. Lanel, “A Study on Graph Theory Properties of On-Line Social Network,” International Journal of Scientific and Research Publications, vol. 10, pp. 267-274, 2020.

[10] G.H.J. Lanel, and E.A.C.T. Sandamal, “A Study on Graph Theory Properties of Constellations,” European Modern Studies Journal [Internet].4:131-143, 2020.

[11] OpenStax, "Circulatory Pathways," in Anatomy and Physiology, Rice University, Creative Commons Attribution 4.0 International License, 2020, [Online], Available: pressbooks.bccampus.ca.

\section{Authors}

First Author - R.B.A.H. Abeyrathne, Department of Mathematics, University of Sri Jayewardenepura, Sri Lanka, araliya.abeyrathne@gmai.com

Second Author - Dr. G.H.J.Lanel, Senior Lecturer (Grade 1), Department of Mathematics, University of Sri Jayewardenepura, Sri Lanka.ghjlanel@sjp.ac.lk. 